\title{
Vegetative State from the Perspective of Islamic Law
}

\author{
Hossein Shamsi Gooshki', Seyyed Hassan Abedian Kalkhoran ${ }^{1 *}$, Seyyed Mohammad \\ Mahdi Ahmadi', Abolfazl Khoshi' ${ }^{2}$ and Hassan Goodarzi ${ }^{2}$ \\ 'Department of Jurisprudence and Principles of Islamic Law, Qom Branch, Islamic Azad University, \\ Qom, Iran; mirhsan4@qom.iau.ac.ir \\ ${ }^{2}$ Faculty of Medicine, Department of Medical Ethics, Baqiyatallah University of Medical Sciences, Tehran, Iran
}

\begin{abstract}
The death of the cerebral cortex is a particular type of brain death that occurs after the destruction of the cerebral cortex (the hemispheres of the brain). It is said that the individual has gone through a vegetative state. This cortex is responsible for controlling voluntary activities of the body. This condition is caused by a coma (anesthesia), and sometimes the individual remains in this state for several years. Although the person looks awake, his/her eyes are open and has some involuntary movements, there is no signs of mental and cognitive function. Moreover, the individual is physically in a state of dementia. Coma is a state in which a person cannot be awakened and does not respond to any stimulation including pain. Generally it lasts few days to a few weeks, after which some patients gradually recover, but some permanently lose all brain function (brain death), while others evolve to a vegetative state (VS). Patients in VS are unconscious and unaware of their surroundings, but they continue to have a sleep-wake cycle and can have periods of consciousness. They are able to breathe spontaneously, retain their gag, cough, sucking, and swallowing reflexes. They often look fairly "normal" to families and friends who hope and pray for their full recovery. Laws and regulations in Islamic countries originate from popular jurisprudence. Therefore, by arguing that the well-known principles of Islam are necessarily legitimate, the phenomenon of vegetative state has been recognized. Jurisprudents have conflicting opinions on brain deaths and these perspectives cannot be considered as a widespread legal basis at the level of macro policy for administrative, medical and judicial affairs. In criminal law, maniac has no criminal responsibility because the punishment is not in line with the purpose of punishment. Consequently, restrictions will be imposed on the patients. Therefore, it can be concluded that a person with vegetative state is compatible with the insanity.
\end{abstract}

Keywords: Brain Death, Cortex, Islamic Law, Vegetative State

\section{Introduction}

For jurists/lawyers, detecting brain death is important due to the fact that the individual with brain death can donate his/her organs. This donation is linked to the respect for the human body after death. Since the only way to use the organs of the dead is through natural death just minutes after death and it is not practically possible according to the medical sciences, physicians give special attention to brain death; a condition in which the organs only go through a kind of vegetative state and the central part of the body (brain) has been broken. Of course, in modern legal interpretation, "brain death is considered as a special condition in the light of new biomedical technologies." ${ }^{\prime}$ In spite of the jurisprudential views on brain death, it does not seem to be a good argument, knowing the irreversibility of a person with brain death. In this regard, other organs have not been taken into consideration, especially the heart and the lungs.

That the brain is the center of command in the body and controls the emotions, feelings as well as voluntary and involuntary acts cannot be sufficient reason for the true death of the person with brain death. The brain alone cannot imply the existence of a living individual, and the heartbeat and respiration depend on the ventilator (artificial respiration). Ventilator is a device that provides breathing for patients who are temporarily or permanently suffering from respiratory problems. In other words, it 
inhales in the lungs and pauses, and exhalation is done spontaneously. On the other hand, it is very difficult to regard an individual with brain death as a living individual, while we know that he/she is gone. In addition to the decline of emotions and perceptions and the lack of voluntary actions, the body of such an individual does not have the ability to perform involuntary actions, because he/she does is not able to breathe. Scientifically speaking, there is no way back.

\section{Legal Views on Brain Death and Vegetative State}

With regard to its cultural and value structure, each country faces the issue of brain death and transplantation differently. Therefore, this issue can be addressed in a specific way and in accordance with the culture. Hence, lawyers and lawmakers have been focusing on the consequences and effects of these issues by seeking to regulate them. Ultimately, have enacted some laws and regulations in this regard. Different countries in the world have the same approach to accept the basis of the problem; that is, the acceptance of brain death after confirming the conditions. This approach allows the countries to perform autopsy and remove the organs to transplant into the body.

In non-Islamic countries, this issue has been accepted. However, the removal of organs of the people with brain death is associated with a kind of extremism. Although the issue of "open-in" consent is still important for the removal of organs and some countries still follow this system, with the increasing need for transplantation in some Western societies, the system "open-out" consent has emerged. This is used as a new solution in the supply of organs of the people with brain death.

The issue of brain death and organ transplantation in the Islamic Republic of Iran is restricted to the medical system and is considered a governmental issue; however, the fact that the jurisprudents have different and sometimes conflicting opinions about brain death and one cannot solely depend on such opinions. Moreover, those ideas and opinions cannot be defined at the level of macro policy for administrative, medical and judicial institutions.

That the brain is the center of command in the body and controls the emotions, feelings as well as voluntary and involuntary acts cannot be sufficient reason for the true death of the person with brain death. The brain alone cannot imply the existence of a living individual, and the heartbeat and respiration depend on the ventilator (artificial respiration). Ventilator is a device that provides breathing for patients who are temporarily or permanently suffering from respiratory problems. In other words, it inhales in the lungs and pauses, and exhalation is done spontaneously. On the other hand, it is very difficult to regard an individual with brain death as a living individual, while we know that he/she is gone. In addition to the decline of emotions and perceptions and the lack of voluntary actions, the body of such an individual does not have the ability to perform involuntary actions, because he/she does is not able to breathe. Scientifically speaking, there is no way back.

If we believe that the return of a person suffering from brain death is rationally impossible without taking into consideration the science and medical statistics, then we must also refrain from burying the dead. The return of the dead, at least on the basis of religious beliefs, is not definitely ruled out. Those who, because of the possibility of maintaining the soul, believe that one with brain death is still alive do not provide a knowledge of the quality of the exodus of the soul from the body. Therefore, given the fact that science does not have definitive ideas about soul, it is preferable not to rely on this unknown criterion in examining the nature of death, although the unknown nature of the soul for human beings is also emphasized in the Quran.?

Overall, brain death seems to be neither definitive death nor life. Rather it is a state resulting from the use of modern biomedical technology and can be justified by the theory of supposed death and not the true death. This situation needs to be further explained in light of medical findings and religious foundations, in particular, one should not overlook the approach to profitability and expediency.

However, given that there is no consensus among the religious scholars on the transplantation of organs from those with brain deaths, it is possible, through cultural foundation, to take the essential steps in promoting the culture of organ donation.

There was a need for inclusive laws in this matter. Hence, in the wake of the ever-increasing proliferation of transplantation and frequent problems, physicians and surgeons demanded legal approval. Finally, this issue was investigated in the legislative area as a bill.

The law "Transplantation of the organs of dead patients or patients whose brain death is certain" was 
adopted in April 17, 2000, without any definition of brain death.

Once obtained written consent from the Ministry of Health and Medical Education, hospitals equipped for organ transplantation can use the healthy organs of the patients whose brain deaths are have been confirmed by the experts. Thus, these organs can be used in patients whose life depends on the transplantation..$^{-3}$

Once the brain death is confirmed, the information, brain death symptoms, the use of non-toxic drugs and the like are ensured. ${ }^{4}$

After the general provisions of the law were approved, its executive code was formulated by the Ministry of Health and Medical Education. It was finally approved by the Cabinet of Ministers in May 2002. The law of "organ transplantation and brain death" has made significant progress in organizing the organs of and has addressed some of the problems and issues that have arisen in this regard.

The legislator had not predicted certain rules on this subject. A bill entitled: "The bill for the organ transplantation of the deceased in special cases", which included the title of people with a certain brain death, was presented to the parliament in May 25, 1997. According to the bill, people with brain deaths are also considered dead, and it is permissible to remove their organs.

This law was not approved in 1995 after the bill was introduced in the parliament's public courtroom. However, it was later passed by the parliament in 2000 and sent to the Guardian Council. 5 The Guardian Council needed to declare its opinion within ten days. Given the fact that this council failed to present its opinion within the deadline, the law was enforced. It was among the few laws that were passed without the involvement of the council.

The majority of the jurists are opposed to cutting off the organs of the people with brain death. In addition, the Guardian Council, which protects religion in the laws of the Islamic Republic of Iran, opposed the parliament. There has been a large number of correspondence between this council and the Islamic Parliament.

\section{Analysis of the Nature of Vegetative State based on Iranian Law}

A person with vegetative state seems to be going to the same situation as that of a person who is insane. So, first, we will analyze the insanity and then generalize to vegetative state.

\subsection{First Clause: The Legal Definition of Insanity}

One of the important issues in medical science, psychiatry, and law is the issue of insanity. Insanity is important in psychiatry is for diagnostic and treatment purposes. Accordingly, it is also legally important in some ways.

\subsection{Types of Insanity}

There are different types of insanity. In psychology, various types of insanity have been identified and several definitions have been presented. Jurists have considered insanity in a variety of different ways. ${ }^{6}$ Insanity is divided into permanent and periodic insanity depending on its continuation. Permanent refers to a state that always accompanies the individual and does not go away. Those who suffer from insanity will never restore and will spend all their lives in a state of insanity. However, periodic insanity is alternate insanity. In this regard, the individual suffers from a state of insanity for some time during life. After a while, it goes away and the individual returns to his/her normal life. In this state, the individual sometimes lives consciously, and sometimes is affected by insanity.

\subsection{Second Clause: Consciousness}

Consciousness is the state or quality of awareness or of being aware of an external object. It has been defined variously in terms of sentience, awareness, qualia, subjectivity, the ability to experience or to feel, wakefulness, having a sense of selfhood or soul, the fact that there is something that it is like to have or be it, and the executive control system of the mind. $\stackrel{-}{\text { At a normal }}$ level of consciousness, the individual is awake and aware. ${ }^{9}$

Reduced consciousness is a condition in which the patient experiences a change in his/her normal consciousness that may vary from navigation disturbance to indifference to all environmental stimuli. Issues of practical concern include how the presence of consciousness can be assessed in severely ill, comatose, or anesthetized people, and how to treat conditions in which consciousness is impaired or disrupted. The degree of consciousness is measured by standardized behavior observation scales A change in the patient's consciousness indicates that the central nervous system has been affected by reduced consciousness for some reason 
Disorders of "consciousness" are characterized by the difficulty in waking the patient which and may originate from an acute damage to the activating system or both of the hemispheres. Coma is the most severe degree of consciousness reduction 10 .

Reduced or altered consciousness: in some cases, the patient's level of consciousness is normal and is awake, but the cognitive function is disturbed. Therefore, it may sometimes result in dementia or some other conditions. Accordingly, although the person is awake, he/she does not have a proper understanding of the world around him.

The study of human brain anatomy and a brief familiarity with it functions play a role in identifying the origin of consciousness and the center of human voluntary action.

\subsection{Third Clause: Brain Anatomy and its Tasks}

Brain is the most complex organ of the human body. Although scientists have done extensive studies about the human brain, further information is required, and not all its actions and mechanisms of action are known. Thus, brain and its anatomy are considered as medical subjects. A brief familiarization with brain and its anatomy can contribute to legal judgments. Brain consists of three main parts: cerebrum, cerebellum and brain stem. Each of these parts has components and performs specific tasks. ${ }^{11}$ It controls most of the activities of the body, processing, integrating, and coordinating the information it receives from the sense organs, and making decisions as to the instructions sent to the rest of the body. The brain is contained in, and protected by, the skull bones of the head.

The cerebral cortex is an outer layer of grey matter, covering the core of white matter. The cortex is split into the neocortex and the much smaller allocortex. The neocortex is made up of six neuronal layers, while the allocortex has three or four. Each hemisphere is conventionally divided into four lobes - the frontal, temporal, parietal, and occipital lobes. The frontal lobe is associated with functions including self-control, planning, reasoning, and abstract thought, while the occipital lobe is dedicated to vision. Within each lobe, cortical areas are associated with specific functions, such as the sensory, motor and association regions. $\frac{12}{}$ Although the left and right hemispheres are broadly similar in shape and function, some functions are associated with one side, such as language in the left and visual-spatial ability in the right. The hemispheres are connected by commissural nerve tracts, the largest being the corpus callosum..$^{13}$

The brainstem consists of the midbrain, the pons, and the medulla oblongata The cerebellum is connected to the brainstem by pairs of tracts.

The cerebrum is connected by the brainstem to the spinal cord. The cerebellum is connected to the brainstem by pairs of tracts. Within the cerebrum is the ventricular system, consisting of four interconnected ventricles in which cerebrospinal fluid is produced and circulated. Underneath the cerebral cortex are several important structures, including the thalamus, the epithalamus, the pineal gland, the hypothalamus, the pituitary gland, and the subthalamus; the limbic structures, including the amygdala and the hippocampus; the claustrum, the various nuclei of the basal ganglia; the basal forebrain structures, and the three circumventricular organs..$^{14}$

The cerebral cortex is divided into two hemispheres and each hemisphere is conventionally divided into four lobes - the frontal, temporal, parietal, and occipital lobes. The frontal lobe is associated with functions including self-control, planning, reasoning, and abstract thought, while the occipital lobe is dedicated to vision. Within each lobe, cortical areas are associated with specific functions, such as the sensory, motor and association regions. Although the left and right hemispheres are broadly similar in shape and function, some functions are associated with one side, such as language in the left and visual-spatial ability in the right.

The frontal lobe can be considered as the most important part of the brain. The main functions of the frontal lobe are to control attention, abstract thinking, behavior, problem solving tasks, and physical reactions and personality. It is responsible for more complex tasks, such as thinking, deciding, and issuing motor commands. Damage to this part disrupts this part of brain activity. In the frontal lobe, the main focus is on intelligence, logic, thinking and, to a certain extent, memory.

The parietal lobe is tasked with controlling sensory perception, including pain, heat, cold and touch. Moreover, spatial vision (orientation) is one of the tasks of this part of the brain. The temporal lobe is the main gateway to entering the peripheral information into the brain and recording it. The temporal lobe controls auditory and visual memories, language, and some hearing and speech. The limbic system refers to a group of neural structures located between the cerebral cortex 
and the hypothalamus. The limbic system exists in all mammals. This system controls emotions, actions, behavior and possibly human memory. $\frac{15}{}$

The brainstem consists of a series of nerves that are responsible for controlling the body's involuntary actions, such as eye movement control, respiratory control, sleep control, cardiovascular and circulatory control, and consciousness. The brainstem is the site of a cranial nerve catheter that is important in determining brain death.

The brainstem, resembling a stalk, attaches to and leaves the cerebrum at the start of the midbrain area. The brainstem includes the midbrain, the pons, and the medulla oblongata. Behind the brainstem is the cerebellum. The cerebrum, brainstem, cerebellum, and spinal cord are covered by three membranes called meninges. The brainstem also contains many cranial nerve nuclei and nuclei of peripheral nerves, as well as nuclei involved in the regulation of many essential processes including breathing, control of eye movements and balance.

Underneath the cerebral cortex are several important structures, including the thalamus, the epithalamus, the pineal gland, the hypothalamus, the pituitary gland, and the subthalamus; the limbic structures, including the amygdala and the hippocampus; the claustrum, the various nuclei of the basal ganglia; the basal forebrain structures, and the three circumventricular organs. The cells of the brain include neurons and supportive glial cells. Overall, the cortex is tasked with performing the acts of thinking, reasoning, and voluntary actions.

This part of the brain is the origin of the decision and the will of man. Therefore, in any case where the cerebral cortex is damaged or its activity stopped, will and voluntary actions of man will be impaired. In this situation, the patient has no will, cannot respond to environmental stimuli and does not understand the surrounding environment. In the vegetative state, the cerebral cortex is unviable. The patient has a regular sleep pattern because the brainstem has a proper activity. According to the neuroscientists, it follows that the origin of the will of the individual and the source of the permission is the cortex. The death of the cerebral cortex is a particular type of brain death that occurs after the destruction of the cerebral cortex (the hemispheres of the brain). This part of the brain controls the voluntary activity of the body. Vegetative state is followed by a coma (anesthesia), and sometimes the individual remains in this state for many years, although the he/ she looks awake, his/her eyes are open and has a series of involuntary movements. Moreover, this individual lacks any cognitive and mental performance. These patients survive severe brain damage for many years and are seemingly conscious, without being able to communicate with others. Moreover, the individual's neurological symptoms are minimized, consciousness and awareness of time and place has declined, and the brain is unable to calculate and judge completely. The power of memory is an important factor for the survival of the autonomy of an elderly man.

\section{Conclusion}

According to the criminal law, the insane person is not accountable for criminal responsibility. ${ }^{16}$ According to the legal matters, restrictions will be imposed on the patient. Therefore, it can be concluded that the situation of one suffering from vegetative state is comparable to that of an insane $e^{\frac{17}{7}}$.

\section{References}

1. Ad Hoc Committee of the Harward Medical School to Examine Definition of Brain Death (A Definition of Ireversible coma), committee of the Harward Medical school 205 J. A. M. A 337 (1968).

2. The Holy Quran.

3. Abbsi, Mahmood, Proceedings of Medical Law, Tehran, Legal. (2001).

4. Larijani, Bagher, Physician and Ethical Considerations, Volume I, Review of Medical Ethics Fundamentals, Tehran, Farda Publications, $1^{\text {st }}$ Edition. (2004).

5. Secretariat of the Guardian Council, Volume 79/11/85. (2004).

6. Hashemi Shahroudi, Sayyed Mahmoud and a group of scholars, Islamic Jurisprudence Encyclopedia on the Religion of the Ahlul Bayt (PBUH), Volume III, $1^{\text {st }}$ Edition, Institute of Islamic Jurisprudence Encyclopedia on the Religion of the Ahlul Bayt (PBUH), Qum. ( 2002).

7. Saberi, Sayyed Mahdi and Mohammadi, Mohammad Reza, A New Approach to Legal Psychiatry, $1^{\text {st }}$ Edition, Teymourzadeh, Tehran. (2005).

8. Ebrahimi Fakhar, Hamidreza; Kohestani, Hamidreza and Baghcheghi, Nayyereh, Loss of Consciousness, $1^{\text {st }}$ Edition, Hayyan Publications, Tehran. (2007).

9. Jennett B, Bond M. Assessment of outcome after severe brain damage: a practical scale. Lancet. 1975;1:480-84. 
10. Plum F, Posner JB. The diagnosis of stupor and coma, $3^{\text {rd }} \mathrm{ed}$. Philadelphia: F. A. Davis, 1980.

11. Monajemi, Alireza, stroke, $1^{\text {st }}$ Edition, Azadmehr, Tehran. (2008).

12. Haeri Rouhani, Seyyed Ali, Nervous Physiology and Endocrinology, 9th Edition, Tehran, Iran. (2007).

13. Khoda Panahi, Mohammad Karim, Physiologic Psychology, $9^{\text {th }}$ Edition, SMT Publications, Tehran. (2011).
14. Barbarestani, Mohammad and Amidi, Fardin, Basic and Clinical Medicine Optical Anatomy, $1^{\text {st }}$ Edition, Noor Danesh Publications, Tehran. (2000).

15. Hall, John Edward, Geyton-Hall Medical Physiology, Translation: Qavami, Yasir and..., Volume II, $1^{\text {st }}$ Edition, Ishraqiyeh, Tehran. (2011).

16. Ardebili, Mohammad Ali, General criminal law, Volume II, $13^{\text {th }}$ Edition, mizan, Tehran. (2006).

17. Iranian Civil Code, Volume II, Article 1211. (1935). 